\title{
Grundsätze zum Umgang mit Forschungsdaten
}

\author{
Die Grundsätze wurden von \\ der Allianz der deutschen Wissenschaftsorganisationen \\ am 24. Juni 2010 verabschiedet \\ Alexander von Humboldt-Stiftung \\ Deutsche Akademie der Naturforscher Leopoldina \\ Deutsche Forschungsgemeinschaft (DFG) \\ Deutscher Akademischer Austausch Dienst (DAAD) \\ Fraunhofer-Gesellschaft \\ Helmholtz-Gemeinschaft \\ Hochschulrektorenkonferenz (HRK) \\ Leibniz-Gemeinschaft \\ Max-Planck-Gesellschaft \\ Wissenschaftsrat
}

Die Grundsätze sind auch abrufbar unter:

http://www.allianzinitiative.de/de/handlungsfelder/forschungsdaten 


\section{„Grundsätze zum Umgang mit Forschungsdaten““}

Die „Grundsätze zum Umgang mit Forschungsdaten“ sind ein Ergebnis im Rahmen der Allianz Schwerpunktinitiative „Digitale Information“, Handlungsfeld 4 „Forschungsprimärdaten“.

Präambel Qualitätsgesicherte Forschungsdaten bilden einen Grundpfeiler wissenschaftlicher Erkenntnis und können unabhängig von ihrem ursprünglichen Erhebungszweck vielfach Grundlage weiterer Forschung sein. Dies gilt namentlich für die Aggregation von Daten aus unterschiedlichen Quellen zur gemeinsamen Nutzung. Die nachhaltige Sicherung und Bereitstellung von Forschungsdaten dient daher nicht nur der Prüfung früherer Ergebnisse, sondern in hohem Maße auch der Erzielung künftiger Ergebnisse. Sie bildet eine strategische Aufgabe, zu der Wissenschaft, Politik und andere Teile der Gesellschaft gemeinsam beitragen müssen. Mit dem Ziel, die Qualität, Produktivität und Wettbewerbsfähigkeit der Wissenschaft zu fördern, verabschiedet die Allianz der deutschen Wissenschaftsorganisationen daher folgende Grundsätze für ein koordiniertes weiteres Vorgehen.

Sicherung und Zugänglichkeit In Übereinstimmung mit wichtigen internationalen Organisationen auf dem Gebiet der Förderung und Durchführung von Forschungsaufgaben ${ }^{(1)}$ unterstützt die Allianz die langfristige Sicherung und den grundsätzlich offenen Zugang zu Daten aus öffentlich geförderter Forschung.

Dabei sind die wissenschaftlichen und rechtlichen Interessen der Forscherinnen und Forscher, der Schutz persönlicher Daten von Probanden, Patienten und anderen von den erhobenen Daten betroffenen Personen sowie weitere Verpflichtungen gegenüber Dritten - etwa Kooperationspartnern - zu beachten. Die Grundsätze der guten wissenschaftlichen Praxis finden Anwendung ${ }^{(2)}$.

Unterschiede der wissenschaftlichen Disziplinen Formen und Bedingungen des Zugangs zu Forschungsdaten müssen gesondert für die jeweiligen Fachdisziplinen unter Berücksichtigung der Art und Weise der Datenerhebung, des Umfangs und der Vernetzbarkeit des Datenmaterials sowie der praktischen Brauchbarkeit der Daten entwickelt werden. Zugleich ist den jeweiligen Lebenszyklen und Nutzungsszenarien der Daten in dem konkreten Forschungsfeld Rechnung zu tragen.

Wissenschaftliche Anerkennung Die Bereitstellung von Forschungsdaten zur weiteren Nutzung ist eine Leistung, die der Wissenschaft als Ganzer zu Gute kommt. Die Allianz ermutigt zur Anerkennung und Förderung dieses zusätzlichen zeitlichen und finanziellen Aufwands.

Lehre und Qualifizierung Für die Forschenden muss ein angemessenes Ausbildungs- und Unterstützungsangebot zum professionellen Datenmanagement bereitgestellt werden, das an den spezifischen Erfordernissen der Disziplinen ausgerichtet ist.

Verwendung von Standards Eine sachgerechte Nutzung von Forschungsdaten setzt voraus, dass sie in standardisierter Form bereitgestellt, dokumentiert und ausreichend mit Metadaten versehen werden. Standards, Metadatenkataloge und Registries sind unter Berücksichtigung fachspezifischer Anforderungen so zu entwickeln, dass auch eine interdisziplinäre Nutzung möglich ist.

Entwicklung von Infrastrukturen Ein nachhaltiges Forschungsdaten-Management stellt vielfältige technische und organisatorische Anforderungen. Diese Anforderungen müssen in Zusammenarbeit von Wissenschaftlerinnen und Wissenschaftlern mit Informationsspezialistinnen und Informationsspezialisten definiert werden. Infrastrukturen sind gemäß diesen Anforderungen zu entwickeln und möglichst von Beginn an in internationale und interdisziplinäre Netzwerke interoperabel einzubinden.

\footnotetext{
1 „The EUROHORCs and ESF Vision on a Globally Competitive ERA and their Road Map for Actions to Help Build It“, ESF Science Policy Briefing 33, June 2008, http://www.esf.org/publications/policy-briefings.html; „OECD Principles and Guidelines for Access to Research Data from Public Funding", OECD 2007.

${ }^{2}$ DFG Denkschrift: Sicherung Guter Wissenschaftlicher Praxis, DFG 1998; http://www.dfg.de/antragstellung/gwp/.
} 